Ann. Zootech., I966, 15 (3), 259-277.

\title{
INFLUENCE SAISONNIÈRE SUR LA PRODUCTION ET LA COMPOSITION DU LAIT
}

\author{
C. DECAEN et M. JOURNET \\ avec la collaboration technique de Y. Manis et B. MARquis \\ Station de Recherches sur l'Élevage des Ruminants, \\ Cintre de Recherches zootechniques et rétérinaires sur les Ruminants, \\ 63 - Theix, près Clermont-Ferrand
}

\section{SOMMAIRE}

Nous avons étudié les variations, d'avril à octobre, de la production de lait et des teneurs en matières grasses et en matières azotées. Pour cela, nous avons tout d'abord décrit l'évolution de ces variables sur des lots d'animaux dans deux troupeaux et pendant cinq années consécutives ; cnsuite nous avons essayé d'analyser l'influence, d'une part du régime alimentaire en comparant des lots d'animaux recevant soit de l'herbe, soit un régime de type hivernal, d'autre part de la conduite d'élevage en comparant des lots d'animaux en stabulation ou au pâturage.

L'évolution de la composition du lait est très comparable dans les 2 troupeaux et d'une année à l'autre : les teneurs en matières grasses et en matières azotées du lait diminuent en mai-juin au lieu d'augmenter avec le stade de lactation ; elles augmentent rapidement en août et surtout en septembre.

L'évolution selon ce schéma général ne semble pas due à des facteurs alimentaires, ni dépendre du mode de vie des animaux (en stabulation ou à l'extérieur). Le facteur responsable doit être de nature climatique; il s'agit sans doute de la durée du jour et de son sens de variation.

\section{INTRODUCTION}

Les nombreux travaux réalisés dans différents pays répartis sur tout le globe (tabl. I et 2) montrent que la quantité et la composition du lait produit par les vaches varient avec la saison. L'influence de la saison telle qu'on l'étudie généralement, résulte des effets combinés de l'alimentation, des facteurs climatiques et du stade de lactation des vaches; il est difficile d'estimer l'influence particulière de chacun 


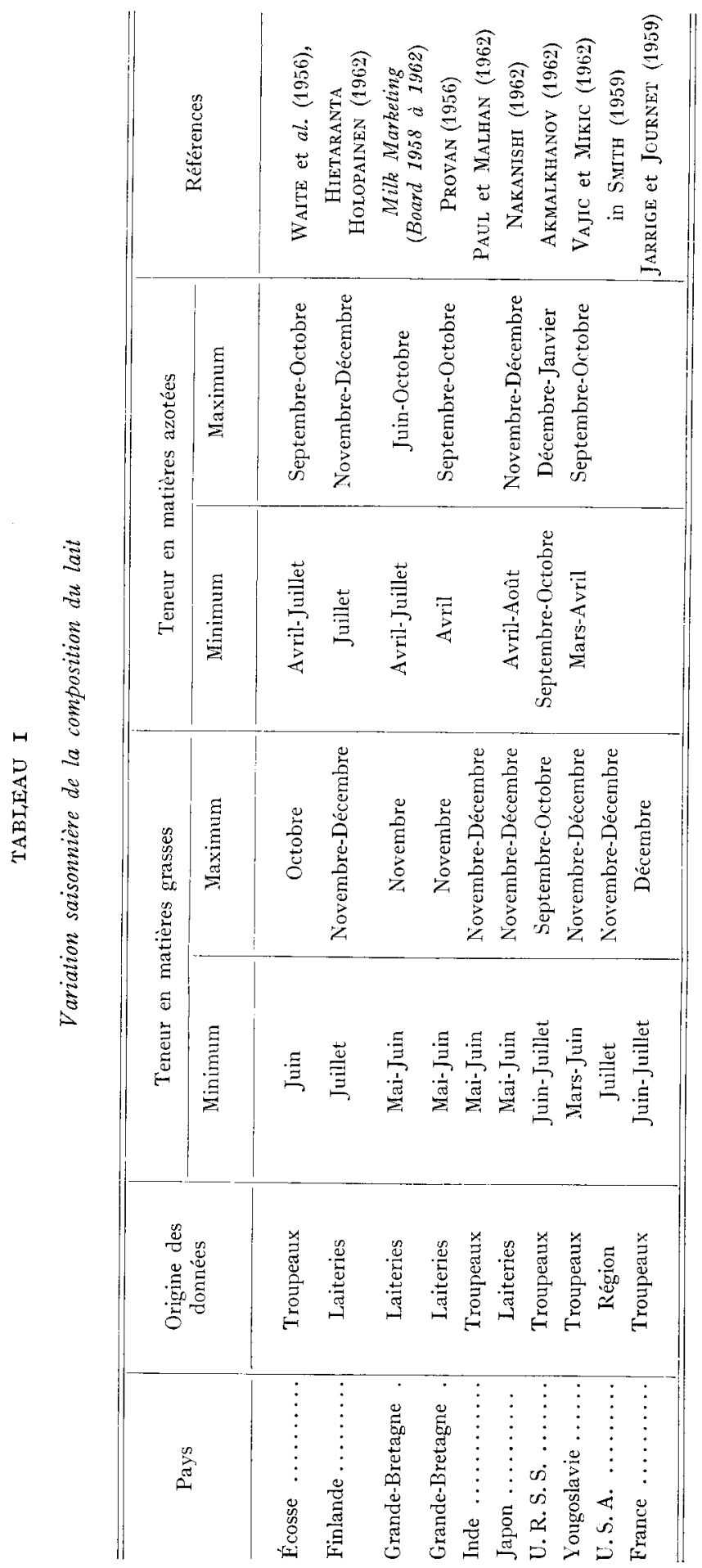


de ces facteurs. L'effet global sur la composition du lait a été montré par certains auteurs (tabl. I), en décrivant 1'évolution saisonnière dans un troupeau, dans une laiterie ou dans un pays ; citons en particulier les travaux de Provan (1956) et du Milk Marketing board (1958-1959-I961-I962) en Grande-Bretagne. Pour tenir compte du stade de lactation, plusieurs auteurs ont observé l'influence de la date de vêlage sur l'évolution de la composition du lait au cours de la lactation (cf. JARRIGE et JoURneT, I959), (PolitiEK, I957). WAITE, WhITE et RobERTSON (I956) utilisent pour leur part, un procédé statistique pour dissocier les influences respectives du stade de lactation et de la saison.

Dans l'ensemble, d'après ces résultats, la teneur du lait en matières grasses est minimum à la fin du printemps et maximum en automne; la teneur en matières azotées présente deux minima, à la fin de l'hiver et à la fin du printemps, et deux maxima au début du printemps après la mise à l'herbe et en automne avant le retour en stabulation. En ce qui concerne la production de lait, on a généralement étudié l'influence de la saison de vêlage sur la production maximum (tabl. 2) ; les valeurs les plus élevées sont observées au printemps et les valeurs les plus faibles en été.

\section{TABLEAU 2}

Infuence de la saison de vélage sur la production maximum de lait

\begin{tabular}{|c|c|c|c|}
\hline Pays & $\begin{array}{c}\text { Saison } \\
\text { favorable }\end{array}$ & $\begin{array}{c}\text { Saison } \\
\text { défavorable }\end{array}$ & Références \\
\hline Finlande.. & Printemps & Automne & BruUn (1928) \\
\hline Finlande... & Hiver-Printemps & Été-Automne & MAKELA (1962) \\
\hline Norvège ... & Hiver-P'rintemps & Été & LAKSESVELA et al. (1952) \\
\hline Allemagne ........... & Iliver & Été & BLAU $(1962)$ \\
\hline Tchéroslovaquie ........ & IIiver & Été-Automne & SUCHANEK (1962) \\
\hline Tchécoslovacuie ........ & Hiver & Automne & KAHOUN (1963) \\
\hline France (Jura) ......... & Hiver-l'rintemps & Fité & AURIOL (1955) \\
\hline France (Yonne) & Printemps & Été & Decaen, Poutols (1965) \\
\hline France (Ile-de-France) ... & Printemps & Été & Decaen, Poutous (1966) \\
\hline Angleterre . . . . . . . . & Printemps & Automne & SANDFRS (1908) \\
\hline Érosse $\ldots \ldots \ldots \ldots \ldots$ & Printemps & Été-Automne & SIKKA $(1950)$ \\
\hline Angleterre $\ldots \ldots \ldots \ldots$ & Printemps & Été & Goocil (1935) \\
\hline États-Lnis $\ldots \ldots \ldots \ldots$ & Automne & Été & PARKER, CNDERWOOI) (195') \\
\hline États-Unis (Géorgie) ..... & Hiver-Printemps & Été & Fosgate, Welch $(1960)$ \\
\hline États-Unis (Géorgic) ..... & Hiver-Printemps & Été & JEE el al. (1961) \\
\hline États-Unis (Illinois) ...... & Printemps & Été & JoHnson ei al. $(1961,1962)$ \\
\hline États-Unis ... & Iiver & Été & CANNON $(1933)$ \\
\hline Inde $\ldots \ldots \ldots \ldots \ldots \ldots$ & IIiver & Automne & Mahesi Dutt, Sukn Bir Singh (1961) \\
\hline
\end{tabular}

La plupart de ces auteurs pensent que ces variations saisonnières de la production et de la composition du lait, dans les pays tempérés, ont des causes alimentaires, bien que souvent les variations de l'alimentation n'aient pu être mesurées. L'influence directe de certains facteurs climatiques (température, humidité, force du vent ...) a été étudiée en chambre climatisée principalement à l'Université du Missouri. I1 est également possible de mettre en évidence assez simplement l'action globale des 
facteurs climatiques et en particulier de la dissocier de celle des facteurs alimentaires, en comparant des lots d'animaux, soumis aux mêmes conditions climatiques mais recevant des rations de nature différente, ou bien en composant des lots de vaches vêlant à des époques différentes de l'année mais recevant durant toute leur lactation une même ration.

L'étude qui suit a été effectuée sur le troupeau de vaches du C. N. R. Z. L.es données recueillies sur ces animaux ont déjà été utilisées précédemment pour étudier l'influence des facteurs saisonniers (JARRIGE, I956; JARRIGE et JouRNET, I959). Dans une première partie, nous avons observé la répétition d'une année à une autre (durant 5 années) et dans 2 stations des courbes d'évolution de la production et de la composition du lait de lots de vaches vêlées en hiver et allant au pâturage d'avril à octobre. Dans une deuxième partie, nous avons essayé d'analyser pendant la même période, l'influence respective de l'alimentation, de la conduite du troupeau et des facteurs climatiques en comparant des lots d'animaux, recevant des rations de nature différente, et alimentés soit en stabulation, soit au pâturage.

\section{ÉTUDE DESCRIPTIVE}

\section{MATÉRIEL, ETT MÉTHODES}

Nous avons observé sur des lots de vaches l'évolution moyenne hebdomadaire, au cours de la saison de pâturage, de la production et de la composition de laits individuels. Pour mettre en évidence l'influence de l'année et du lieu, nous avons essayé d'éliminer l'influence du stade de lactation des animaux. Pour cela, nous avons composé chaque année, de $195^{8}$ à $19^{6} 2$, un lot de 10 vaches à la Station de Jouy et un lot de 8 vaches à la Station de la Minière qui se trouvaient en moyenne en fin du $2^{\mathrm{e}}$ mois de lactation au moment du début de l'expérience, en avril. Ainsi au cours de la saison de pâturage, les animaux se sont toujours trouvés dans la phase décroissante de la lactation, entre le premier et le huitième mois après la mise bas.

\section{Alimentation et conduite des troupeaux}

Les vaches de $\mathrm{n}^{0}$ de lactation compris entre 2 et 8 étaient de races Firisonne, Normande et Pie Rouge : 39 et 24 Firisonnes, , o et 12 Normandes, 1 et 4 Pies rouges, respectivement dans les troupeaux de Jouy et de La Minière.

Le régime alimentaire était composé avant la mise à l'herbe de foin et d'ensilage distribués à volonté et de $20 \mathrm{~kg}$ de betteraves. Insuite les animaux pâturaient des prairies temporaires composées le plus souvent d'une graminée et d'une légumineuse. Ia nature et le mode d'exploitation de ces prairies ont été précisés dans une étude précédente (DEMARQLILIY, I963). Les besoins des animaux ont été assurés par un apport d'aliment concentré dont la quantité a été calculée d'après la production individuelle de lait et l'apport connu de la ration d'hiver ou estimé du pâturage.

Les 2 Stations de Jouy et de La Minière ne sont éloignées que de $8 \mathrm{~km}$, mais leur lieu d'implantation et leur microclimat sont assez différents : les prairies de Jouy sont situées dans une vallée alluvionnaire abritée, alors que celles de La Minière sont sur un plateau à limon, très découvert. En outre, l'alimentation des 2 troupcaux n'a pas été rigoureusement la même ; alors que les prairies de La Minière étaient surtout de Dactyle, de Luzerne ou de Dactyle et Luzerne associés. à Jouy le dactyle, prédominant en 1958, a progressivement été remplacé par du Ray-grass et de la Fétuque des prés. Les apports complémentaires de concentré furent moins abondants à La Minière qu'à Jouy ; de plus, dans cette dernière station, les prairies furent arrosées en cas de sécheresse estivale. La conduite du troupeau, le mode d'exploitation des prairies et les conditions de traite furent semblables dans les deux stations; cependant à La Minière, certains pâturages sont très éloignés du lieu de traite et les parcours effectués chaque jour par les animaux sont beaucoup plus longs qu’à Jouy. 
De $195^{8}$ à i962, les méthodes d'alimentation ont changé ; les apports d'aliments complémentaires, en particulier ceux de concentré, ont augmenté. lin 1958 , une vache de $600 \mathrm{~kg}$ recevait $500 \mathrm{~g}$ de concentré par kilogramme de lait produit au-dessus de $24 \mathrm{~kg}$, en avril-mai lorsque le pâturage était de très bonne qualité, et au-dessus de $14 \mathrm{~kg}$ en août avec un pầturage de qualité moyenne ; en 1962 , ces limites de production n'étaient plus que de 20 et $12 \mathrm{~kg}$.

Le passage de la stabulation hivernale au pâturage a eu lieu entre le I I et le 24 avril selon les

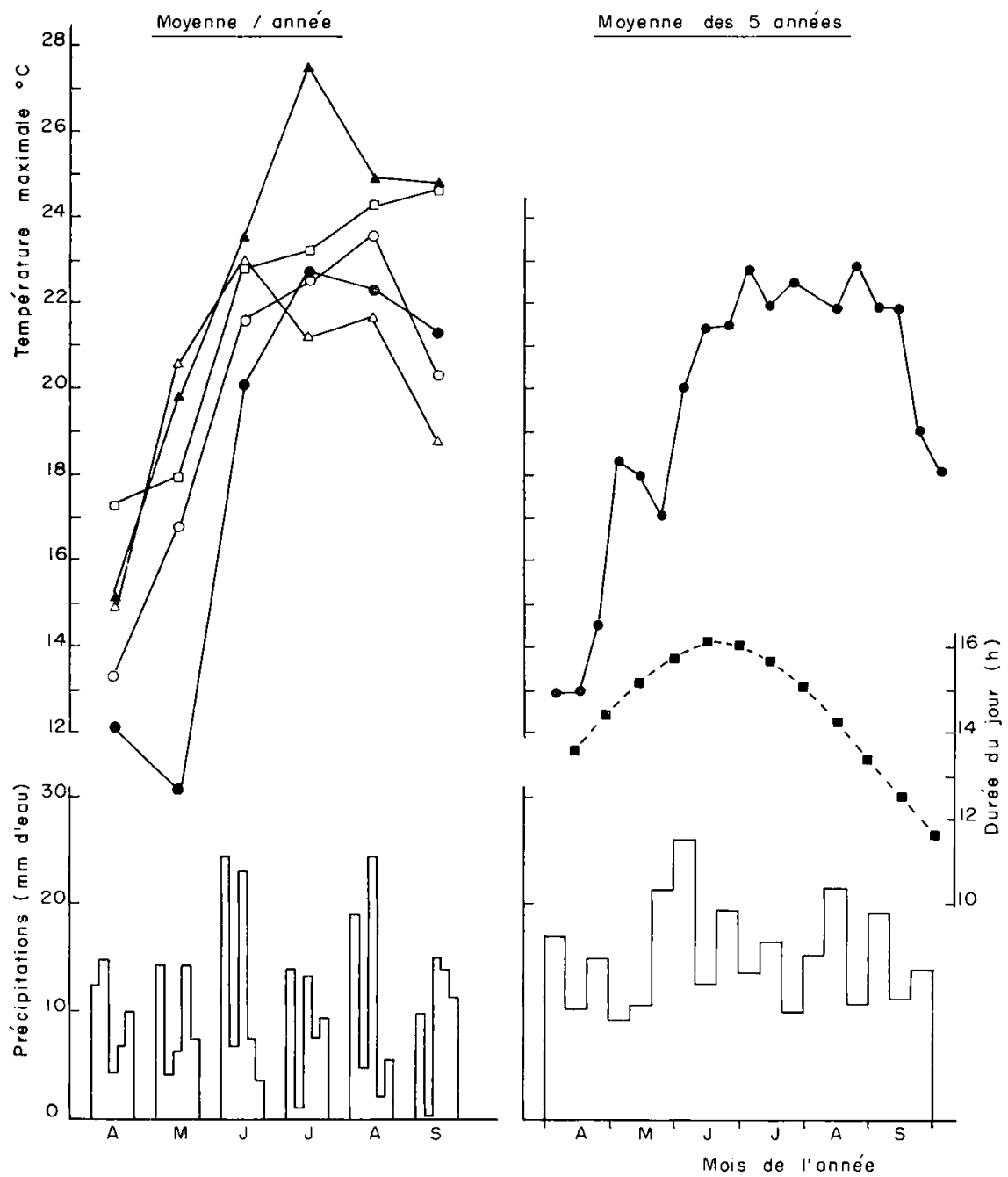

FIf. I. - Évolution des données méléorologiques d'auril à octobre, chaque année de 1958 a 1962, et en moyenne pendant les 5 années

- et I - I958

A et $2-1959$

$\Delta$ et $3-1960$

$\square$ et $4-196 \mathrm{I}$

O et $5-1962$

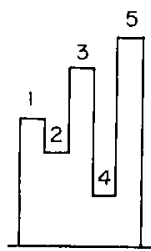


années : au foin, à l'ensilage et aux betteraves, on a substitué l'herbe très progressivement, en augmentant chaque jour, pendant environ Io jours, la durée du pâturage et en rêduisant les apports de fourrages conservés et d'aliment concentré.

\section{Données recueillies}

\section{Production laitière.}

Le lait de chaque vache a été pesé à chaque traite du matin et du soir. La teneur en matières grasses a été mesurée chaque jour par la méthode Gerber sur un échantillon de lait moyen, représentatif des deux traites; nous avons calculé, chaque semaine, la quantité moyenne journalière de matières grasses sécrétée (moyenne de 7 jours). La teneur en matières azotées a été mesurée chaque semaine par la méthode Kjedahl jusqu'en I960, puis par la méthode au noir amido (SCHOBER et HETzeL, 1956), sur un échantillon de lait moyen représentatif de 4 traites consécutives, du milieu de la semaine. La mesure par cette dernière méthode a été faite à la Station de Recherches laitières de Poligny. A partir de cette teneur et de la production de lait moyenne des 4 traites correspondantes, nous avons calculé chaque semaine, la quantité moyenne journalière de matières azotées sécrétées.

\section{Données météorologiques.}

Des nombreuses données météorologiques recueillies, nous avons retenu : la température maximale, la température minimale et les précipitations. L'évolution saisonnière movenne (par année) de ces différentes variables est illustrée par la figure $\mathrm{r}$. La période chaude s'étend̀ de la mi-juin au début de septembre, mais les températures maximales journalières dépassent rarement $30^{\circ} \mathrm{C}$ en juillet ou août. Les précipitations sont en moyenne bien réparties sur toute la saison de pâturage, mais on doit noter que les pluies de juillet et août sont souvent orageuses. Cependant, cette évolution varie d'amplitude d'une année à l'autre : si $195^{8}$ et 1960 furent froids et humides, 1959 au contraire fut sec et chaud.

\section{Méthodes d'étude}

Nous avons effectué une étude descriptive de l'évolution hebdomadaire des productions de lait de matières grasses et de matières azotées et des teneurs du lait en matières grasses et en matières azotées, de la première semaine d'avril à la dernière semaine de septembre ; nous avons tracé les courbes d'évolution moyenne :

a) pour chaque étable, chaque année : Io vaches à Jouy, 8 vaches à La Minière,

b) pour chacune des deux étables,

c) pour l'ensemble des vaches.

I’uis nous avons analysé statistiquement l'influence de l'étable et de l'année sur la quantité totale de lait produite au cours de la saison de pâturage. Les niveaux de production initiaux des lots d'animaux étant différents d'une étable à l'autre et même d'une année à l'autre, nous avons effectué une analyse de covariance pour éliminer l'influence du niveau de production maximum individuel; afin de classer les moyennes estimées par lot représentatif d'une année et d'une étable, nous avons utilisé le test de Tuckey (SNEDECOR, 1959).

\section{RÉSUITATS}

\section{Évolution moyenne}

L'évolution moyenne dans les deux stations sur l'ensemble des cinq années de la production de lait, de matières grasses, de matières azotées et des teneurs en ces deux constituants est illustrée par le tableau 3 et la figure 2.

- A la mi-avril au moment où, en moyenne, les vaches passent de la stabulation au pâturage, nous retrouvons (DEMARQUILLY, JouRNET, Ig62) l'évolution suivante : la quantité de lait cesse de diminuer puis augmente légèrement et se stabilise pendant environ 3 semaines. La teneur en matières grasses dans le même temps, augmente de façon très importante de $2,6 \mathrm{~g} \mathrm{p}$. I ooo, et celle en matières azotées de I,5 g p. I ooo, mais celle-ci de façon beaucoup plus progressive que la teneur en 
matières grasses. En conséquence à la mise au pâturage, les quantités de matières grasses et de matières azotées produites s'accroissent.

- A partir du mois de mai, la quantité de lait baisse plus ou moins régulièrement jusqu'au début d'octobre ; elle diminue plus rapidement en mai et d'août à septembre,

TABLEAU 3

Évolution saisonnière moyenne des quantités de lait, de matières grasses el de matières azotées produites par 90 vaches au total

(étables de Jouy et de La Minière de 1958 à 1962)

\begin{tabular}{|c|c|c|c|}
\hline \multirow[b]{2}{*}{ Mois } & \multicolumn{3}{|c|}{$\begin{array}{c}\text { Variations relatives mensuelles en } \mathrm{p}, 100 \\
\left(\frac{n^{\text {leme }} \text { semaine du mois }-1 \text { re semaine }}{n} \times 100\right)\end{array}$} \\
\hline & Quantité de lait & $\begin{array}{l}\text { Quantité de } \\
\text { matičres grasses }\end{array}$ & $\begin{array}{l}\text { Quantité de } \\
\text { matières azotées }\end{array}$ \\
\hline Avril . & -3 & +1 & +1 \\
\hline Mai .. & -13 & -20 & -15 \\
\hline Juin & -10 & $-\quad 9$ & -10 \\
\hline Juillet & -7 & -10 & -5 \\
\hline Août. & -14 & -11 & -12 \\
\hline Septembre $\ldots \ldots \ldots \ldots \ldots \ldots$ & -20 & -10 & -9 \\
\hline Moyenne..$\ldots \ldots \ldots \ldots \ldots$ & -11 & -10 & -9 \\
\hline
\end{tabular}

et plus lentement début juin et fin juillet-début août. Mais ce sont les teneurs en matières grasses et en matières azotées qui présentent l'évolution la plus importante ; elles décroissent, très rapidement d'abord, puis plus lentement, jusqu'à la fin juin. Ainsi, exception faite de l'effet de la mise au pâturage sur la teneur du lait en ces deux constituants,celles-ci diminuent de la mi-avril à la fin juin, alors qu'à ce stade de la lactation, elles devraient augmenter.

- En juillet, la teneur en matières grasses augmente dans la première moitié du mois puis redécroît ; la teneur en matières azotées se stabilise. Puis à partir du mois d'août, les teneurs en ces deux constituants s'accroissent très rapidement, respectivement de 5,0 et $4,8 \mathrm{~g}$ p. I ooo.

Dans l'ensemble, les teneurs en matières grasses et en matières azotées évoluent de la même façon excepté en juillet, mais l'amplitude de variation de la teneur en matières grasses est plus importante. Les productions de matières grasses et de matières azotées décroissent très rapidement en mai-juin, puis régulièrement ensuite de Io p. roo par mois.

D'une matière générale, la sécrétion đu lait ainsi que celle des matières grasses et des matières azotées est donc stimulée en avril et non en mai-juin. A partir du mois d'août, la sécrétion du lait est de nouveau freinée, mais cette fois, celle des matières grasses et des matières azotées ne l'est pas; on observe alors un enrichissement spectaculaire du lait en matières grasses et en matières azotées. 


\section{Différences entre années}

Fait remarquable, l'évolution de la composition du lait s'est répétée de façon semblable selon le schéma décrit, chaque année, dans les deux troupeaux, à 1'exception de 1959 à La Minière (fig. 3). En particulier, les teneurs en matières gras-

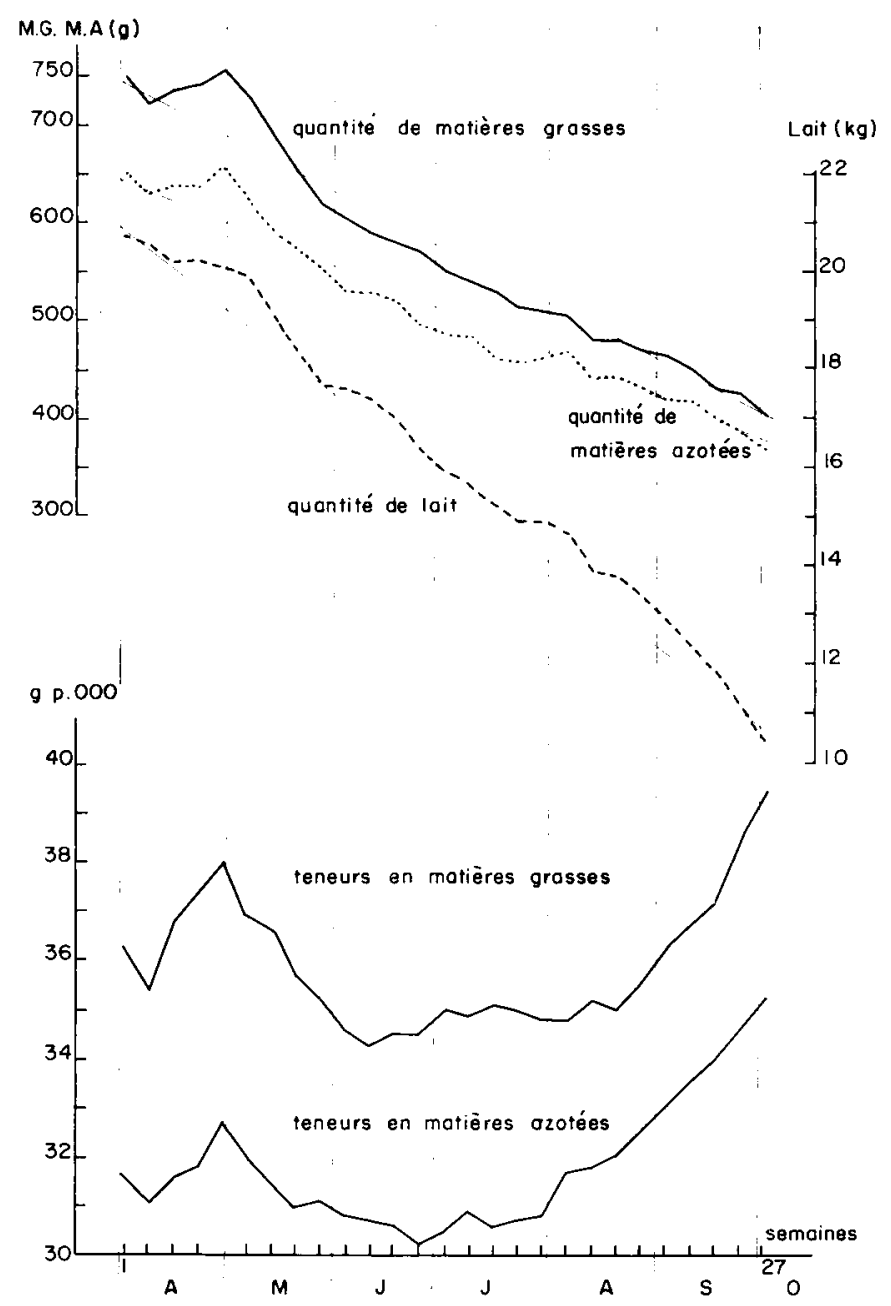

FIG. 2. - Evolution moyenne des quantités de lait, de matières grasses et de matières azotées, de la leneur en malières grasses et matières azolées d'avril à oclobre

ses et en matières azotées ont présenté un minimum qui s'est presque toujours situé en juin et juillet. La décroissance a souvent été rapide en mai et la croissance rapide en septembre. Cependant dans le détail, la forme des courbes d'évolution a varié. $\mathrm{L}_{\mathrm{a}}$ a variabilité hebdomadaire est importante; celle de la teneur en matières grasses est supérieure à celle de la teneur en matières azotées, comme le montrent les courbes d'évolution au cours de la saison de pâturage. L'amplitude de variation a également varié d'une année à l'autre. 

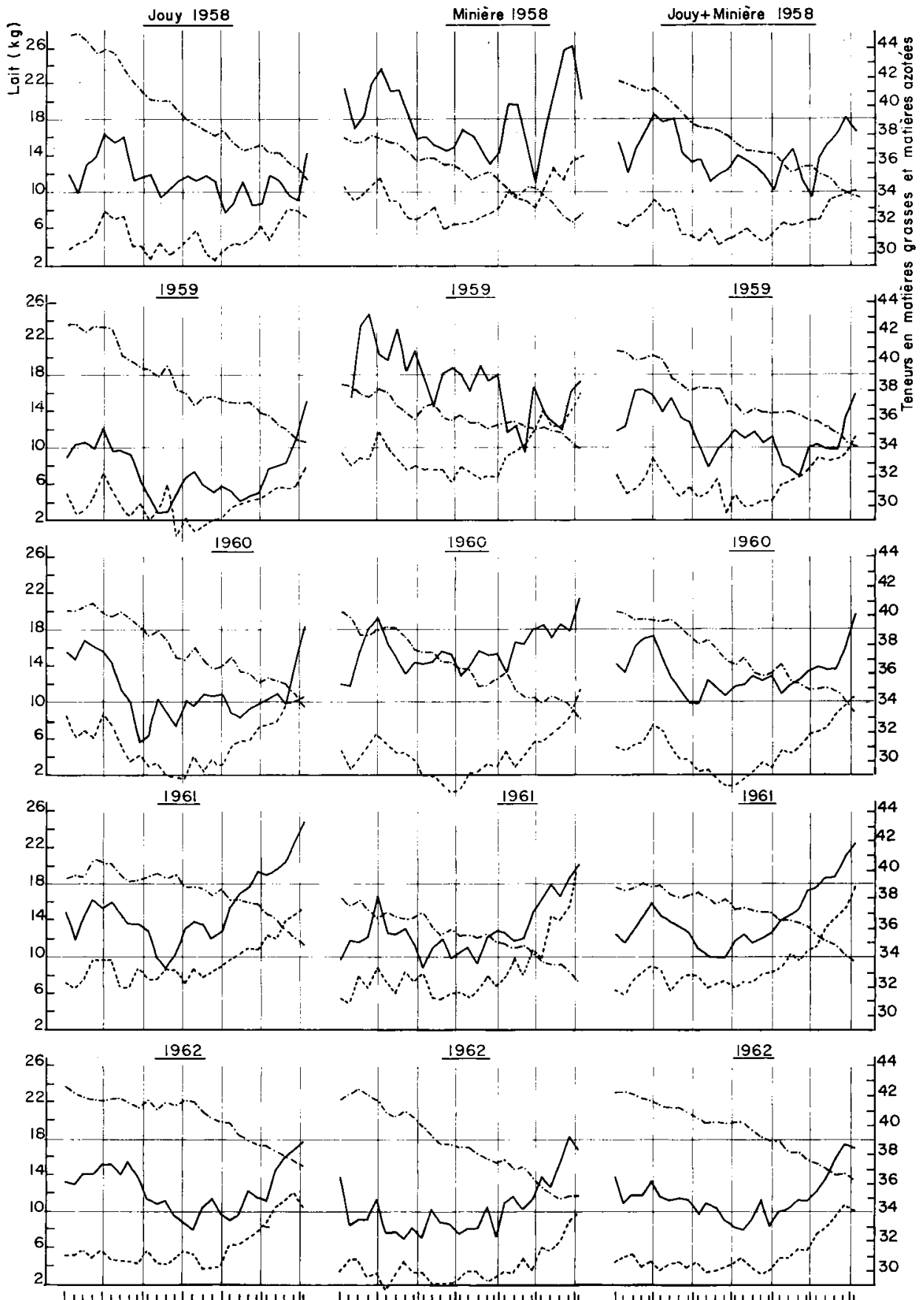

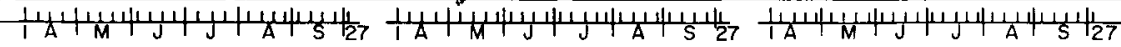

FIG. 3. - Comparaison entre années et entre stations

.. - Lait, — teneur en matières grasses, .... teneur en matières azotées 
Nous nous sommes demandés si la température ne pouvait pas expliquer certaines des différences d'évolution de la teneur en matières grasses observées entre années sur la moyenne des $\mathrm{I} 8$ vaches ( 8 à Jouy + Io à La Minière). La diminution de la teneur en matières grasses en mai et son augmentation en septembre coïncident avec des variations en sens inverse de la température. Cependant, si on relie les variations hebdomadaires de la teneur en matières grasses à celles de la température, on n'observe aucune liaison. De même, si on analyse les résultats année par année, la liaison entre la teneur en matières grasses et la température (moyennes hebdomadaires) n'est significativement négative que deux années sur cinq, et elle est due uniquement au sens de variation inverse en début et en fin de saison de pâturage. Mais ces liaisons ont été établies à partir de coefficients de corrélation simple et sont, de ce fait, difficiles à interpréter

Une des différences les plus marquées entre années est la remontée passagère de la teneur en matières grasses en été. Il semble bien que celle-ci corresponde aux premières grandes chaleurs du début de l'été et qu'elle ait surtout existé les années les plus chaudes, lorsque la température moyenne a dépassé $20^{\circ}$.

Bien que présentant peu de liens avec l'influence de la température, il est important de remarquer que les variations hebdomadaires de la teneur en matières grasses ont été inverses de celles de la quantité de lait quelle que soit la période considérée, début, milieu ou fin de la saison de pâturage. Pour ces 3 périodes, les coefficients de corrélation ont été respectivement de $0,20,0,40$ et 0,86 .

\section{Différences entre troupeaux}

Les vaches du troupeau de Jouy ont produit en moyenne plus de lait ( 25 p. Ioo) mais un lait plus pauvre en matières grasses $(2,0 \mathrm{~g} \mathrm{p}$. I 00o) et en matières azotées (0,5 g p. I ooo) Mais nous retrouvons sur l'ensemble de la saison de pâturage une évolution semblable dans les deux troupeaux des teneurs en matières grasses et en matières azotées, et qui s'est répétée presque toutes les années (fig. 3 et 4). Il existe cependant quelques différences systématiques : l'amplitude de variation de la teneur en matières grasses est plus importante à Jouy qu'à La Minière. A Jouy, les valeurs minimales observées au milieu de juin sont inférieures de $2,5 \mathrm{~g} \mathrm{p}$. I ooo à celles du début avril avant la mise à l'herbe, alors qu'à La Minière, elles sont à peine plus faibles. En outre, à Jouy, en été, la teneur en matières grasses remonte de façon passagère, présentant deux minima fin juin et début août; alors qu'à La Minière, elle présente un palier minimum de la mi-juin au début d'août. La teneur en matières azotées a souvent décru plus rapidement en mai à l'étable de Jouy.

Les différences dans les courbes d'évolution de la production laitière entre les deux troupeaux se sont accrues au fur et à mesure des années de $195^{8}$ à r 962 . A Jouy, la quantité de lait s'est de mieux en mieux maintenue au cours du printemps et du début de l'été. Une analyse de variance a permis de montrer que l'influence de l'année (après élimination de l'influence du niveau de production maximum individuel) est statisquement significative à Jouy alors qu'elle ne l'est pas à La Minière (tabl. 4). A Jouy, la production totale théorique de lait a augmenté régulièrement de $16,5 \mathrm{p}$. Ioo en 5 ans, soit de $630 \mathrm{~kg}$ sur une durée de pâturage de 27 semaines. Cela vient essentiellement de ce qu'à Jouy, les méthodes d'exploitation des pâturages ont pu être améliorées par l'irrigation notamment. 
TABLEAU 4

Influence de l'étable et de l'année sur la quantité totale de lait produite au cours de la saison de pâturage

Les productions estimées ont été obtenues après correction de l'influence du niveau de production maximum individuel, par analyse de covariance (les moyennes qui ne sont pas statistiquement différentes les unes des autres au seuil de probalité $5 \%$ sont marquées de la même lettre $a, b$ ou $c$ )

\begin{tabular}{|c|c|c|c|c|}
\hline \multirow{2}{*}{ Années } & \multicolumn{2}{|c|}{ Données non corrigées } & \multicolumn{2}{|c|}{$\begin{array}{c}\text { Estimées d'après l'analyse } \\
\text { de covariance }\end{array}$} \\
\hline & Jouy & La Minière & Jouy & La Minière \\
\hline $1958 \ldots \ldots \ldots \ldots$ & 3617 & 2343 & $2901^{a}$ & $2888^{a}$ \\
\hline $1959 \ldots \ldots$ & 3254 & 2561 & $2985^{a}$ & $3043^{a b}$ \\
\hline $1960 \ldots \ldots \ldots \ldots$ & 3180 & 2669 & $3218^{b c}$ & $2821^{a}$ \\
\hline $1961 \ldots$ & 3248 & 2363 & $3308^{c}$ & $2938^{a}$ \\
\hline $1962 \ldots \ldots$ & 3834 & 3297 & 3533 & $3034^{a b}$ \\
\hline Moyennes & 3425 & 2467 & 3189 & $29 t_{13}$ \\
\hline
\end{tabular}

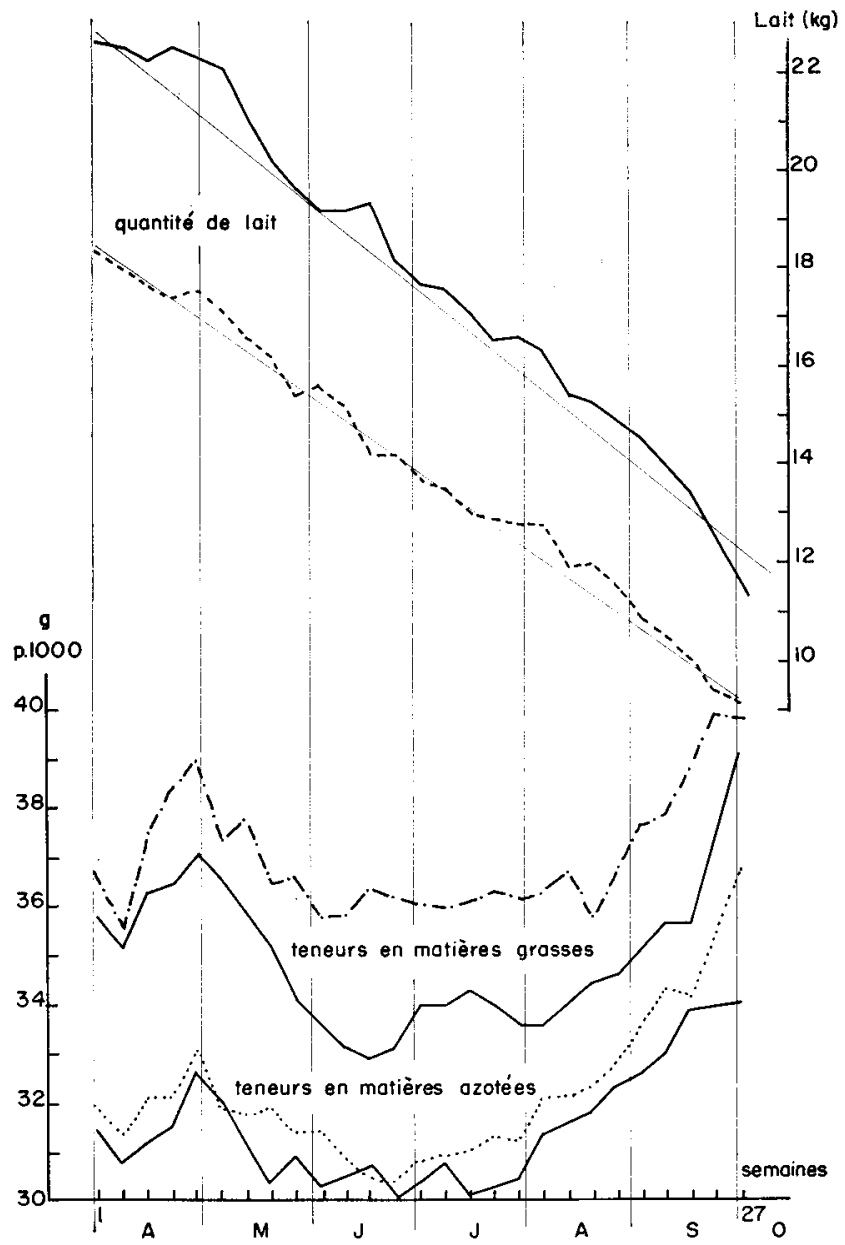

Fig. 4. - Évolution comparée de la quantité de lait et des teneurs en matières grasses et matières azotées d'avril à octobre, dans les stations de Jouy (trait plein) et de la Minière (trait pointillé) 


\title{
ÉTUDE EXPÉRIMENTALE
}

\author{
MATÉRIEL ET MÉTHODES
}

Nous avons comparé l'évolution au cours de la saison de pâturage de la production de lait et des teneurs en matières grasses et en matières azotées de deux lots de vaches :

- conduits de façon différente, soit au pâturage, soit en stabulation, mais consommant la même herbe,

- ou alimentés de façon différente, soit avec un régime de type hivernal, soit avec de l'herbe coupée, ou au pâturage.

Dans chaque essai, les deux lots ont été composés avant le début de l'expérience en appariant les vaches, selon les critères suivants : âge, stade de lactation, niveau de production, composition du lait.

\section{Comparaisons effectuées}

\section{Pâturage et ration hivernale à l'auge.}

En 1962, à l'étable de Jouy, un lot de 8 vaches a reçu en stabulation du foin, et des betteraves remplacées en mai par des drèches fraîches de brasserie, et un aliment concentré. Le lot de vaches appariées pâturait des prairies temporaires. Ces deux lots ont été suivis d'avril à juillet ; à cette date deux autres lots ont été reconstitués afin d'éliminer les animaux dont le stade de lactation dépassait le $8^{\mathbf{e}}$ mois, et comparés jusqu'en octobre.

En I963, à l'étable de Jouy, un lot de 8 vaches, alimentées à l'auge avec du foin et un aliment concentré, a été comparé d'avril à juillet à un lot pâturant des prairies temporaires. Comme en 1962 et pour la même raison, deux autres lots de 9 vaches cette fois-ci ont été constitués fin juin et suivis jusqu'en octobre.

En I964, à l'étable de Jouy, un lot de 9 vaches a reçu à l'auge du foin de luzerne long et du foin de luzerne condensé (broyé et aggloméré) distribués à volonté et un aliment concentré ; il a été comparé d'avril à octobre à un lot de vaches de La Minière, qui a reçu de la luzerne verte à l'auge.

\section{Pâturage et herbe distribuée à l'auge.}

En I960, nous avons comparé d'avril à août, deux lots dans chacune des étables de Jouy et de La Minière : un lot a pâturé des prairies temporaires, l'autre lot a reçu à l'auge l'herbe coupée de ces mêmes prairies.

En I96I, à La Minière nous avons comparé deux lots dans les mêmes conditions que ci-dessus, d'avril à octobre.

Les données sur la quantité de lait et la teneur en matières grasses recueillies sur ces lots d'animaux ont été présentées partiellement dans une étude précédente (DEmarouilly, 1963).

\section{Alimentation et conduite d'élevage}

Les vaches au pâturage ont été alimentées et conduites comme dans l'étude précédente. Celles en stabulation ont reçu la ration de type hivernal (chacun des aliments étant distribué une fois par jour) ou bien l'herbe distribuée quatre fois par jour; les quantités consommées individuelles ayant été mesurées chaque jour, l'apport d'aliment concentré a été ajusté chaque semaine aux besoins de chaque animal. Les vaches ont eu accès pendant quatre heures, à une aire d'exercice en plein air. Elles ont été traites dans les mêmes conditions, à la même heure.

\section{RÉSULTATS}

\section{Comparaison entre l'alimentation à l'herbe et l'alimentation de type hivernal (fig. 5)}

A la mise à l'herbe, les variations de la production et de la composition du lait sur le lot au pâturage correspondent à celles que nous avons rapportées précédemment : la teneur en matières grasses a augmenté rapidement dès la première semaine ; 
la production de lait et la teneur en matières azotées ont augmenté progressivement au cours des trois premières semaines. Ces variations n'ont pas été observées avec le lot recevant le régime hivernal. Sur ce lot cependant, on a observé que la distribution de drèches en remplacement des betteraves à partir de la $2^{\mathrm{e}}$ semaine de mai avait le même effet sur la production de lait et la teneur en matières grasses que la mise à l'herbe sur les autres animaux.

A partir du milieu de mai, la production de lait a évolué parallèlement dans les deux lots en I962 et I964. En I964, cependant elle s'est mieux maintenue avec le lot recevant le régime hivernal. En I963, par contre, avec ce régime, elle a diminué plus rapidement et surtout plus irrégulièrement; ce qu'on peut expliquer car le foin a changé plusieurs fois.

La teneur en matières grasses a évolué dans l'ensemble de façon parallèle dans les deux lots en I 962 avec un minimum d'été très accentué (diminution de $5 \mathrm{~g}$ p. I 000 de mai à juillet) ; ce qui se retrouve également en I963. Le décalage observé à partir
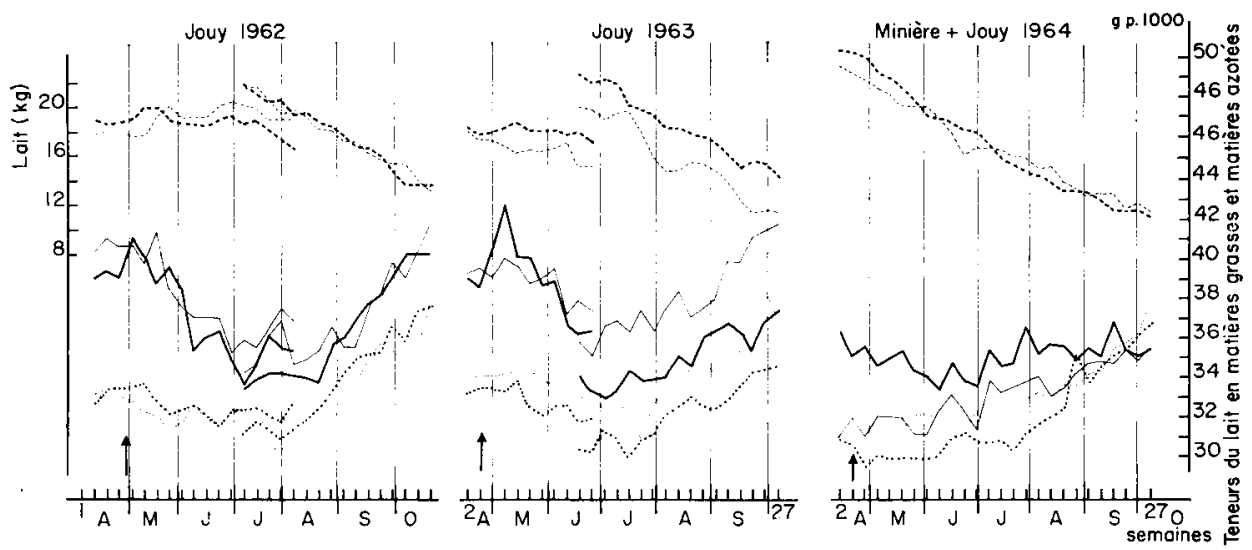

FIG. 5. -- alimentation à l'herbe (trait fort) et alimentation de type hivernal (trait fin)

- - Lait, - teneur en matières grasses, .... $\uparrow$ Mise à l'herbe

de fin juin dans les courbes d'évolution correspond aux changements de lots d'animaux. Par contre, en I964, l'effet dépressif des mois de juin et juillet ne s'est pas fait sentir sur les vaches recevant le régime de type hivernal. Les mêmes remarques peuvent être faites en ce qui concerne l'évolution comparée de la teneur en matières azotées dans les deux lots au cours des 3 années. En I 963 , cependant l'évolution a été quelque peu différente de mai à fin juin.

En I964, pour interpréter les différences d'évolution de la composition du lait des deux lots, il faut rappeler que ceux-ci ont été suivis dans deux étables différentes et que les deux régimes à base de fourrages conservés ou de fourrage vert ont été particuliers : le premier lot a reçu comme fourrage uniquement du foin de luzerne en majorité sous forme broyée et agglomérée, le deuxième lot a reçu de la luzerne verte coupée, au lieu d'un pâturage essentiellement sur graminées comme dans les deux autres comparaisons. En outre, les vaches recevant de la luzerne verte n'ont 
pas toujours été alimentées rigoureusement selon leurs besoins ; elles ont en particulier été sous-alimentées en fin de $\mathrm{I}^{\mathrm{er}}$ cycle de croissance de 1'herbe.

Cette restriction faite, il semble donc que quel que soit le régime des animaux, la production et la composition du lait évoluent selon le schéma décrit dans la première partie et que la nature de l'alimentation ne semble avoir une influence qu'en avril, à la mise à l'herbe, en particulier lorsque les animaux pâturent des graminées.

\section{Comparaison entre une alimentation d'herbe en stabulation et au pâturage}

L'évolution de la production et de la composition du lait a été parallèle, dans l'ensemble, pour les vaches au pâturage et pour celles qui ont reçu l'herbe coupée en stabulation (fig. 6).

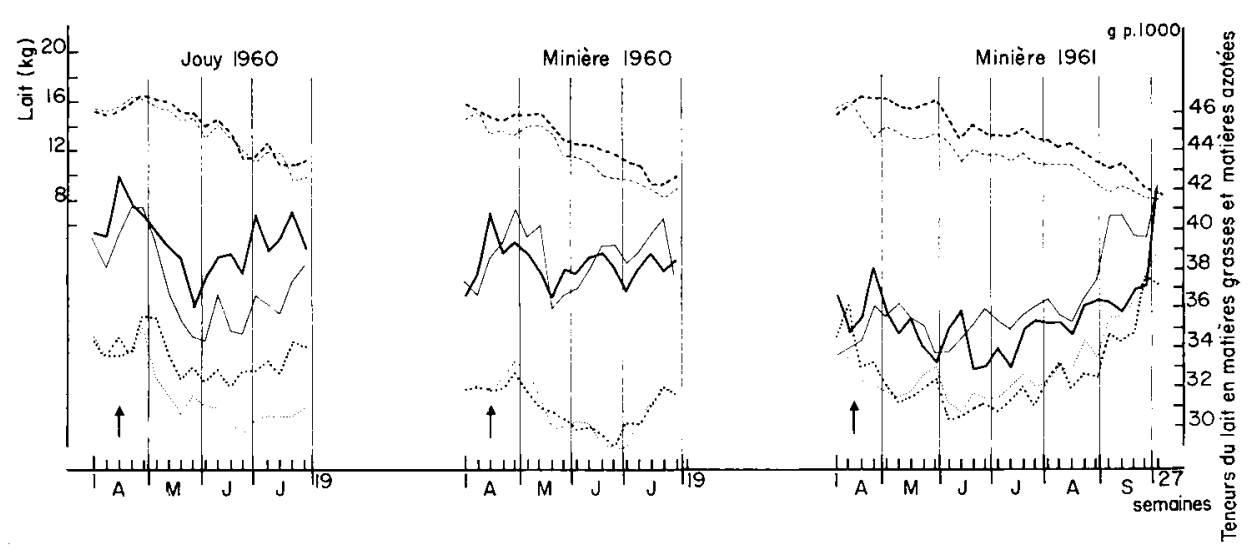

FIG, 6. - Palurage (trait fort) et herbe en stabulaiton (trait fin)

- . - Lait, __ teneur en matières grasses, .... teneur en matières azotées

En I960, aussi bien à Jouy qu'à La Minière jusqu'au début mai, la production de lait et les teneurs en matières grasses et en matières azotées augmentent, c'est la période de la mise à l'herbe. Au cours du mois de mai, c'est-à-dire au cours du premier cycle de végétation de la plante, la production laitière et surtout les teneurs du lait diminuent : 4,5 à $6 \mathrm{~g} \mathrm{p}$. I ooo pour les matières grasses, $2,5 \mathrm{~g}$ à $3 \mathrm{~g} \mathrm{p}$. I ooo pour les matières azotées; à partir de juillet, ces deux variables commencent à remonter. En I96I, à La Minière, on retrouve au cours du printemps l'influence stimulante de la mise à l'herbe puis l'influence inhibitrice de la fin du premier cycle (les teneurs en matières grasses et en matières azotées diminuent respectivement de 3,5 et $3 \mathrm{~g}$ p. I ooo) ; ensuite jusqu'en octobre, les teneurs en matières grasses et en matières azotées augmentent respectivement d'environ $7,5 \mathrm{et} 7 \mathrm{~g}$ p. I ooo.

En conclusion, quel que soit le mode de conduite des animaux, à l'étable ou an pâturage, l'évolution saisonnière de la production laitière reste semblable au schéma décrit précédemment. 


\section{DISCUSSION}

L'évolution de la composition du lait que nous observons au cours de la saison de pâturage confirme les résultats antérieurs obtenus sur le même troupeau (JARRIGE, I956-I957 ; JARRIGE et JOURNET, I959) ; elle est semblable à celle observée dans de nombreux pays des hémisphères Nord et Sud (tabl. I) ; son amplitude de variation est très importante et surtout elle est très reproductible. Cette évolution s'effectue en effet selon le même schéma d'une année à l'autre et dans les deux troupeaux : d'avril à juillet, elle a lieu à contre-courant de l'évolution physiologique ; pendant cette période, les teneurs en matières grasses et en matières azotées décroissent au lieu de croître avec l'avancement de la lactation. Ensuite en août et surtout en septembre, elles croissent plus rapidement qu'elles ne le feraient sous la seule influence du stade de lactation.

La composition du lait a évolué selon ce schéma aussi bien avec les vaches en stabulation qu'au pâturage, et quel que soit le type d'alimentation (herbe ou ration hivernale), les animaux ayant par ailleurs été alimentés selon leurs besoins. Ceci nous autorise à penser que le facteur principal de variation ne doit être recherché ni dans le mode de conduite du troupeau, ni dans les variations de la qualité du pâturage, ni dans le niveau des apports alimentaires, contrairement à l'hypothèse de Waite et al. (I956) et Rook (I96I). Ces résultats sont en accord avec ceux de WITT (I955) qui n'a pas réussi à éviter la baisse de la teneur du lait en matières grasses, après la mise à l'herbe, en distribuant aux vaches des aliments complémentaires de nature très diverse.

\section{Facteurs alimentaires.}

Les facteurs alimentaires n'ont eu qu'un rôle temporaire ou secondaire. A la mise à l'herbe, comme l'un d'entre nous l'a rapporté antérieurement (DFMARQUILLY et JoURnet, I962) les teneurs du lait en matières grasses et en matières azotées s'accroissent; ainsi dans cette étude, les valeurs de la fin avril au pâturage ont été en moyenne supérieure de 2,6 à I,6 g pour I ooo respectivement à celles du début du mois en régime hivernal. Ces accroissements n'ont pas été observés sur les vaches qui continuaient de recevoir à l'auge la même ration hivernale, par contre, ils l'ont été sur des animaux recevant à l'auge de l'herbe coupée. Dans ce cas, c'est donc bien 1'ingestion d'herbe qui est responsable de l'enrichissement du lait à la fois en matières grasses et en matières azotées. En outre, C. DEMARQUILLY (I963) a montré sur les mêmes troupeaux que la nature du pâturage avait une influence importante sur la composition du lait.

Le niveau des apports énergétiques de la ration à certaines périodes peut expliquer facilement les variations parallèles de la teneur en matières azotées du lait. En particulier, il semble que fin mai-début juin, les vaches aient été sous-alimentées car la production de lait a diminué de plus de Io p. Ioo par mois ; ce qui s'est produit lorsqu'elles ont pâturé des graminées en fin de premier cycle. Or, à cette période, la teneur en matières azotées a baissé rapidement. Les dernières années en I96r et I962, les vaches ont reçu une meilleure alimentation complémentaire; la quantité de lait 
s'est mieux maintenue et la teneur en matières azotées n'a pas diminué comme les années précédentes. D'une façon générale, la teneur en matières azotées a varié d'une semaine à la suivante parallèlement à la quantité de lait, en particulier d'avril au début de juillet lorsque l'évolution générale de la quantité de lait et de la teneur en matières azotées s'est faite dans le même sens.

\section{Facteurs climatiques.}

Parmi les facteurs climatiques, la température ne peut pas non plus expliquer l'évolution générale de la composition du lait; elle ve semble avoir agi que de façon temporaire, bien qu'il soit difficile dans cette étude descriptive d'isoler son action de celle des autres facteurs. En effet, si certaines années en I958 et I959, l'évolution de la teneur en matières grasses au cours de la saison de pâturage a été inverse de celle de la température, ce ne fut pas le cas les autres années. En outre, il n'a existé aucune relation entre les variations hebdomadaires de la teneur en matières grasses et de la température. Il semble seulement que la légère remontée de la teneur en matières grasses observée certaines années, fin juin, soit due aux premières chaleurs. Inversement pendant cette période, la teneur en matières azotées ainsi que la quantité de lait ont diminué. On peut penser que ces variations de la composition du lait consécutives à une élévation de la température sont dues indirectement à une sousalimentation. C'est ce qui a été supposé par WorSTEL L et BROBY (I953), HANCOCK (I954) Findlay et Beakley (I954) et ce qui a été prouvé par Wayman et al. (I962).

Le fait que la température n'ait pas joué un rôle important n'est pas étonnant, si on en juge par les travaux des chercheurs de l'Université du Missouri. Ceux-ci ont montré que la zone de confort des vaches laitières se situe entre $\mathrm{o}^{\circ} \mathrm{C}$ et $\mathrm{x} 6^{\circ} \mathrm{C}$; mais ce n'est qu'au-dessus de $27^{\circ}$ pour les Holstein et $29^{\circ} 5$ pour les Jersey et les Brown Swiss que des températures constantes provoquent une baisse de la production de lait et des teneurs en matières azotées et en lactose (RAGSDALE et al., I949I95O-I95I ; COBBLE et HERMAN, I95I). En soumettant des vaches non plus à des ambiances à température constante, mais à des cycles de température variable allant de $2 \mathrm{I}^{\circ} \mathrm{C}$ à $3^{\circ} \mathrm{C}$ ou de $-\mathrm{I} 2^{\circ} \mathrm{C}$ à $4,5^{\circ} \mathrm{C}$, MERILAN et BOwER (I959) n'observent une influence notable de telles températures sur la production laitière et sur la teneur en matières grasses qu'avec les vaches Holstein; en particulier, quand des cycles chauds succèdent sans transition à des cycles froids.

Il semble bien que le facteur susceptible d'expliquer l'évolution générale de la composition du lait d'avril à octobre soit la durée du jour ou tout au moins son sens de variation. En effet, l'évolution saisonnière de la composition du lait s'est répétée dans des conditions diverses d'alimentation et de climat; dans ces différentes conditions, la durée du jour est sans doute le critère du milieu dont l'évolution est la plus répétable. Et surtout les minima des teneurs du lait en matières grasses et en matières azotées ont lieu toujours à la même date, c'est-à-dire au solstice d'été quand la durée du jour cesse de croître puis commence à diminuer.

D'autre part, on sait que chez les bovins, il existe un contrôle photopériodique des activités sexuelles et hypophysaires (OrTavant, Maulíon et THibaulT, I964); or, la sécrétion du lait étant elle-même sous contrôle hypophysaire, il est logique de 
penser que la durée du jour peut agir sur la composition du lait par l'intermédiaire de l'hypothalamus et de 1'hypophyse. Cependant, avant de retenir cette hypothèse, il est nécessaire de recourir à une expérimentation.

Rę̧u pour publication en aồt 1966.

\section{SUMMARY}

\section{SEASONAL EFFECTS ON PRODUCTION AND COMPOSITION OF MILK}

Seasonal variations in yield of milk and its fat and protein contents were studied in daily individual samples between April and October.

In one study curves showing changes were drawn for groups of 8 to Io cows in 2 herds during 5 consecutive years. The cows calved at the end of winter and were fed on a winter ration, then were on pasture from mid-April.

In another the effects of feeding, management and climate were analyed. For that purpose a comparison was made between groups of cows (I) which ate either herbage or a winter ration, and (2) which were fed on herbage indoors or were on pasture.

I. In the first, descriptive, study there were 90 cows, and in the second, experimental, study there were I04. Milk from each cow was weighed at each milking, fat content was estimated once a day and protein content once a week.

2. On going to pasture yield of milk, and particularly of fat and protein increase; fat content increase rapidly, by $2.6 \mathrm{~g}$ per $\mathrm{kg}$, and protein content more slowly, by $\mathrm{r} .5 \mathrm{~g}$ per $\mathrm{kg}$.

3. From the beginning of May these contents fall to a minimum at the end of June, then they increase rapidly in August and particularly in September. As a result, between the beginning of April, before going to grass, and the end of June, 3 months later, the milk becomes less rich, on average by $2.0 \mathrm{~g}$ per $\mathrm{kg}$ for fat and by $1.4 \mathrm{~g}$ for protein. These changes are the opposite to those which usually take place at this stage of lactation.

4. The changes in composition of milk repeat the same pattern when the cows have been on a winter ration instead of grass or when they have been fed indoors on grass instead of grazing.

5. It was not possible to show any relation between changes in temperature and changes in fat content.

6. The change in milk composition was the same in each year, whatever the climatic conditions, and in each herd, regardless of feeding and management.

7. Thus length of day was the only constant factor which had a cycle of change similar to that seen in fat and protein contents of milk.

8. The curves showing changes in yield and composition of milk did differ, however, in detail and in amplitude. Among nutritional factors, plane of feeding showed some variation parallel to that for yield of milk and protein content during the pasture season, and between years. Among climatic factors, the highest temperatures observed in July seemed to have caused an increase in fat content.

\section{RÉFÉRENCES BIBLIOGRAPHIQUES}

AkMalkilanov S. H., J962. Variation in composition of milk at the hot and cool seasons in Uzbekistan. XVIe Congr. Internat. Laiterie. Section I : 1, 265-269.

Aurior P., r955. Influence du mois de vêlage sur la production laitière des vaches Pie Rouge de l'Est dans le Jura. Ann. Zootech., 4, $189-200$.

BLAU G., I962. Untersuchungen über den Verlauf der Laktationskurve. II. Bezrehungen zwischen Haltevemogen und Laktationsertrag und der Einfluss von Laktationsdauer und Kalbmonat auf die Form der laktations kurve. Züchlungskunde, 33, 373-393.

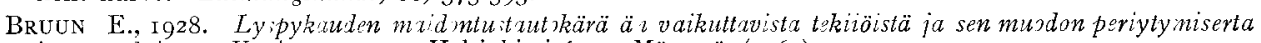
itäsumalaissesa Karjassa, I2 I p. Helsinki cité par MÄkeLÄ (1962).

Cannon C. Y., I933. Seasonal effect on yield of dairy cows. J. Dairy Sci., 16, Ir-r 5 .

Cobble J. W., Herman H. A., I95 I. The influence of environmental temperature on the composition of milk of the dairy cows. Mo. Agric. Exp. Sta. Res. Bul. 485 . 
Decaen C., Poutous M., ig65. Phase ascendante de la courbe de lactation chez la vache laitière. Ann. Zoolech., 14, I $35^{-1} 43$.

Decaen C., Poutous M., I 966. Évolution de la production laitière de la vache au cours des 2 premiers mois de la lactation. Ann. Zootech., sous presse.

Demarquilly C., Journet M., 1962. Variations de la composition du lait à la mise à l'herbe. XVIe Congr. Internat. Laiterie, Section, I : 1, 33-48.

Demarquilly C., I963. Influence de la nature du pâturage sur la production laitière et la composition du lait. Ann. Zootech., 12 69-104.

Findlay J. L., Beakley W. R., 1954. Environmental physiology of farm mammals. In Progress in the Physiology of farm animals, vol. 1 , chap. 6 Hammond. Buttersworth Scientific Publications, p. 252-298.

Fosgate O. T., Welch H. K., 1960. Effect of certain environmental factors upon milk production in Georgia. J. Dairy Sci., 43, 442 (Ábstr.)

Goocir M., I935. An analysis of the time change in milk production in individual lactations. J. agric. Soc., 25, $7 \mathrm{I}$.

HANCOCK J., I954. Direct influence of climate on milk production. Dairy Sci. Abstr., 16, 89.

Hietaranta M., Holopainen P., ig62. Der Ginfluss des Wetters während der Weideperiode auf das Verhältnis Fett. Protein in der Milch. XVIe Congr. Internat. Laiterie. Section I : 1, I37-143.

JARRIGE R., I956. Variations de la teneur en matières azotées des laits individuels. VII e Congr. InternatZoolech., Madrid 5 .

JARrige R., Journet M., i 959. Influence des facteurs alimentaires et climatiques sur la teneur en matières grasses du lait. Ann. Nutrit. Aliment., 13, A $233^{-2} 78$.

Johnson K. R., Ross R. H., FourT D. L., Hibbs R. A., Ig6r. Causes of variation in the solids not fat and butterfat contents of cow's milk.

Johnson R. V., TOuch Berry R. W., ig62. Influence of month of calving on lactation milk yield. $57 / h$ Anim. Meet. Amer. Dairy Sci. Ass. Cell.g? Park, Maryland p. 56.

Kahoun J., r963. Analyza vzestupné fâze laktace, rocni doby teleni a ekonomiky chovı dojnic z klediska perzistence laktacni Krivky. Zivocisna Vyroba, 8, 9I-I Io.

LAKSESvela B., Homb T.,BREIREM K., I952. Milk yield and profit of milk production at different seasons of calving. III. Opstadt. Norg. Landbruk ø hogsk Beretn. For Forsøk, in Dairy Sci. Abstr., I954, p. Io4.

Lee J. E., Fosgate O. T., Carmon J. L., ig6r. Some effects of certain environmental and inherited influences upon milk and fat production in dairy cattle. J. Dairy Sci., 44, 296-299.

Mahesi Dutt. Sukh Bir Singh. I96I. Effect of the season of calving on the milk yield, peak yield and lactation period in Hariana Cattle. Indian J. Dairy Sci., 14, I61-165.

MÄKELÄ A., I962. The lactation curve of the cow at the ascending phase. Maataloust. Aikakausk, 34, 173I86.

Merilan C. P., Bower K. W., I959. Influence of daily environmental temperature cycles on composition of cow's milk. Mod. agric. Exp. Stat. Res. Bul. 645.

National Milk Recrrds, r965. Southerna Edition. Milk Marketing Board. Thames Jitton Survey, p. I 2.

Nakanishi T., 1962. On some factors influencing the fat/protein ration in milk, in Japan. XVIe Congr. Internat. Laiterie. Seciton I : 1, I $29^{-1} 3^{6}$.

Ortavant R., Mauléon P., Thibault C., ig64. Photoperiodic control of gonadal and hypophysical activity in domestic animals. Ann. N. Y. Acad. Sci., 117, 157-192.

Parker J. B., Underwood P. C., I954. Care and management of dairy cows. U.S.D.A. Bull. I470.

PaUl M. T., Mahlan R. T., 1962 Studies on the composition of the milk of Indians Animals. XVIe Congr. Internat. Laiterie., Section I : 1, $225^{-2} 35$.

Politiek R., i957. De invloed van erfeligkheid en milieu op de samenstelling van de melke bij Friese koeien en də praktische mogelijkheid van selecte op het eiwitgeholte. Thi se doctorat. Drukkerij Folkertsma Drachten, p. I 75 .

PRovan A. L., I956. Variations in the composition of milk from individual animals and herds. Report of Milk Marketing Board.

Ragsdale A. C., Worstell D. M., Thompson H. J., Brody S., ig49. Influence of temperature, 50 to $0^{\circ}$ and $50^{\circ} \mathrm{F}$ to $95^{\circ} \mathrm{F}$, on milk production, feed and water consumption and body weight in Jersey and Holstein cows. Mod. agric. Exper. Stat. Res. Bul. 449. Environmental Physiology VI.

Ragsdale A. C., Thompson H. J., Worstell D. M., et Brody S., i950. Milk production and feed and water consumption reponses of Brahman, Jersey and $H_{0 l s t}$ in cows to changes in temperature, $50^{\circ}$ to $105^{\circ} \mathrm{F}$ and $50^{\circ}$ to $80^{\circ}$ F. Mod.agric. Exp. Stat. Res. Bul. 460 . Environmental Physiology IX.

Ragsdale A. C., Thompson H. J., Worstell D. M., Brody S., I95I. Influence of increasing temperature $40^{\circ}$ to $105^{\circ} \mathrm{F}$, on milk production in Brown Swiss cows, and on feed and water consumption and body weight in Brown and Brahman cows and heifers. Mod. agric. Exp. Stat. Res. Bul. 47, Environmental Physiology XII.

Rook J. A. F., I96r. Variations in the chemical composition of the milk of the cow. Dairy Sci. Abstr., 23, $25 \mathrm{I}-258$.

Rosetti C., JARrige R., 1957. Études sur les variations de la richesse des constituants azotés du lait de vache. IV. Relations entre la teneur en protéines et le taux butyreux. Ann. Zootech., 2, I23-152. 
SANDERS H. G., I928. The variations in milk yields caused by season of the year, service, agre and dry period and their elimination. Part. I II. J. agric. Sci., 18, 46-67.

Sciober R., Hetzer. II. F., 1957. Vergleich der photometrischen Milchproteinbestimmung mittels Amidoschwarz ro B mit titrimetrischen Eiweissbestimnung durch Thrombacid. Milchwissenschaft, 12, 12-14.

SIKkA L. C., I950. A study of lactation as affected by heredity and enviromment. J. Duiry Sci., 17, 23 I-252.

Sмrтh V. R., 1959. Physiology of lactation. Constable and Company Ltd. London. 5 édition, p. 2 I 9.

SNedecor G. W., 1959. Stalistical methods applied to exprimenls in agriculiure and biology. The Iowa State

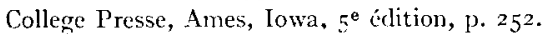

SuchaneK 33., ig62. Hodnoceni a význain tvaru laktačnich Křivek dojnic Zivocisna Vyroba 7, 549-562.

VAJIC B., MiKIC F., ro62. Korrelationen zwischen den Eiweiss und Fettgehalt und der Milchmenge bei Kühen unter mangelhafter Hinährung und ungünstigen Stallungsbedingungen. XVe Congr. Internal. Lailerie, Secion I : 1, 236-246.

WAite R., WIITE J. C. D., ROBERTSON A., 1956. Variation in the chimical composition of milk with particular reference to the solids not fat. J. Dairy Res., 23, 65-8I.

Wayman O., Johnson H. D., Merilan C. P., Berry I. L., ig6z. Effect of cd lib'un or forcefeeding of two rations on lactating cows subject to temperature stress. J. Dairy Sci., 45, I472-I 478 .

Wiтt M., i955. Arch. Tierennähr., 5, i 66.

Worstell D. M., Broby S.. I95.3. Comparative physiological reactions of European and Indian cattle to changing temperature. Ma. agric. Exp. Sta. Res. Bul. $n^{\circ}$ i 5 Envirommental Physiology XX. 